Novel Thiosalamo Ligand as a Remarkably Stable $\mathrm{N}_{2} \mathrm{~S}_{2}$ Salen-type Chelate and Synthesis of a Nickel(II) Complex

Supporting Information

Shigehisa Akine and Tatsuya Nabeshima*

Department of Chemistry, University of Tsukuba

Tsukuba, Ibaraki 305-8571, Japan. 


\section{ESI-mass spectrum of [Ni(tsalamo)]}



Figure S1. ESI mass spectrum of [Ni(tsalamo)].
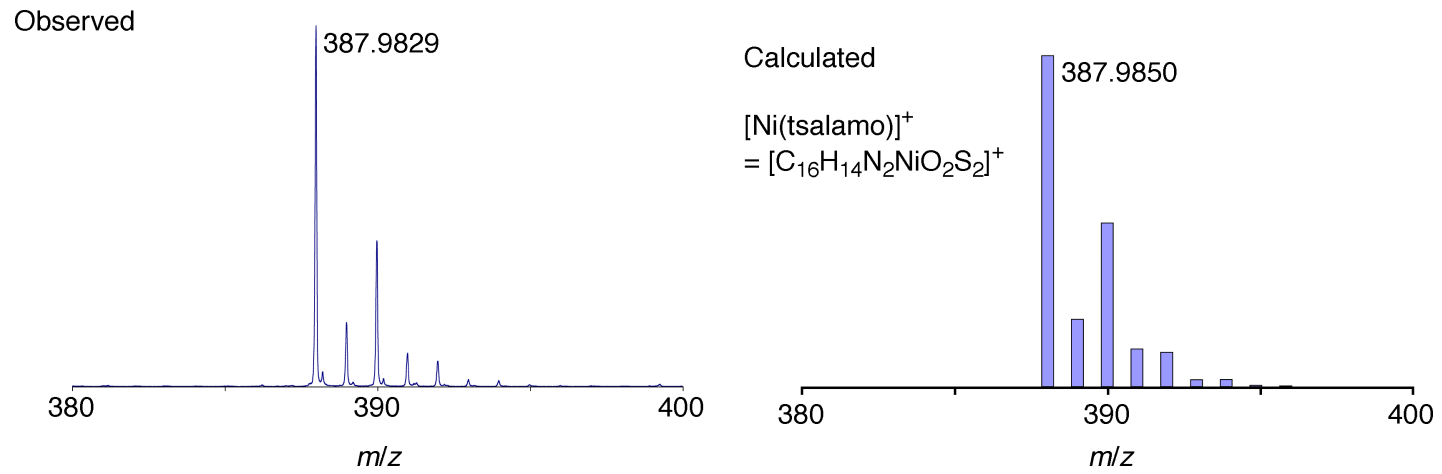

Figure S2. Observed and calculated isotope patterns of [Ni(tsalamo) $]^{+}$. 